\title{
A Novel Search for Free Quarks Produced in Heavy-Ion Interactions
}




\title{
LETTER OF INTENT TO THE SPSC
}

October 3, 1997

\section{A NOVEL SEARCH FOR FREE QUARKS PRODUCED IN HEAVY-ION INTERACTIONS}

\author{
CERN $^{1}$-IRVINE 2 -LANL ${ }^{3}$-LBL ${ }^{4}$-SCHAFER 5 -SLAC 6 \\ CD Hendricks ${ }^{5}$, GL Shaw ${ }^{2}$, EL Garwin $6 *, \mathrm{~K}$ Lackner $^{3}, \mathrm{H} \mathrm{Matis}{ }^{4 *}, \ldots \ldots . .1$ \\ Spokesmen: CD Hendricks and GL Shaw \\ Contactman: To be added 1
}

GENEVA

1997

\section{CERN}

2 Physics Dept. University of California, Irvine CA 92717 USA

3 Los Alamos National Lab, Los Alamos, NM USA

4. Lawrence Berkeley Lab, Berkeley CA USA

5 Schafer Corporation, Livermore, CA 94550 USA

6. SLAC, Stanford CA 94305 USA

* Indicates consultant 


\title{
A Novel Search for Free Quarks Produced in Relativistic Heavy-Ion Interactions
}

\begin{abstract}
We propose a novel search for free anti-diquarks ( $\underline{\mathrm{uu}} \Rightarrow$ ) $\mathrm{Q}$ with electric charge $-4 / 3$ produced in heavy-ion collisions induced by the ultra relativistic heavy-ion beam $\mathrm{Pb}$ beam at CERN. Stopping the $\mathrm{Qs}$ in a dewar of liquid deuterium, will allow the Qs to catalyze d-d fusion by rapidly forming ddQ molecules. The resultant products stop in a short distance, by the ionization of the $\mathrm{D}_{2}$. When the ions and electrons recombine, photons will be emitted which can be detected by photomultiplier tubes. If a heavy-ion beam bunched in time is provided, any Qs stopped in the dewar should continue this catalysis process long after any secondary beam interaction particles have left the dewar.
\end{abstract}


We propose a novel way to search for free quarks produced in heavy-ion collisions by the present $\mathrm{Pb}$ beam at the CERN, see Fig. 1. This proposal relies heavily on the glow model [1-3] of broken QCD in which stable antidiquarks ( $\underline{\mathrm{u} u} \Rightarrow$ ) $\mathrm{Q}$ with electric charge $-4 / 3$ are produced. These $\mathrm{Qs}$, if stopped in liquid deuterium, will catalyze the fusion reactions [4] at a rate of roughly $10 \% / \mathrm{s} / \mathrm{Q}[5]$ and continue for some time:

$$
\begin{aligned}
(\mathrm{ddQ})_{\text {molecule }} \rightarrow{ }^{4} \mathrm{He}+\mathrm{Q}+23.9 \mathrm{Mev} & \text { i) } \\
{ }^{3} \mathrm{He}+\mathrm{n}+\mathrm{Q}+3.3 \mathrm{MeV} & \text { ii) } \\
\mathrm{t}+\mathrm{p}+\mathrm{Q}+4.0 \mathrm{MeV} & \text { iii) }
\end{aligned}
$$

with (1i) being the dominant reaction [5]. The key component of our detector is a dewar of liquid $D_{2}$ in which the Qs would stop and proceed to catalyze fusion (until the $\mathrm{Q}$ reaches a wall and gets trapped in a high $\mathrm{Z}$ atom). The light produced by the stopping ${ }^{4} \mathrm{He}$ nucleus and $\mathrm{Q}$ in a series of reactions (1i) would be detected by photomultiplier tubes (PMTs). Analysis of the time history of data output from the PMTs would allow us to rule out various backgrounds, e.g., $\mu$ catalyzed fusion [6-7] (which will also help calibrate our detection efficiency). Although this proposal rests on speculative assumptions, there is a unique signal from the catalyzed fusion of the deuterium atoms and the consequences of successful detection are profound.

The search for free quarks had a substantial number [8-15] of experiments in the early 1980's, but has been quiet since then until the search for free quarks using the new high throughput automated Millikan type apparatus [16] being developed at SLAC [17]. The proposed accelerator experiment should be considered as complementary to the new bulk matter search.

There is an impressive body of experimental and theoretical literature supporting quark confinement. Theoretical investigations have shown that unbroken non-Abelian gauge theories confine the charges of the localsymmetry groups. However, it is not possible to determine definitively 
from present theoretical and experimental results if the exact local symmetry in nature is $\mathrm{SU}(3)^{\text {color }} \times \mathrm{U}(1)^{\mathrm{em}}$, in which case particles with color are confined. Models have been presented in which $\mathrm{SU}(3)^{\text {color }}$ is spontaneously broken and color is not an exact local gauge symmetry $[18,1]$. Here, free quarks (or diquarks) could be produced in certain experiments and yet not violate present experimental and theoretical constraints.

For the purposes of this proposal, we will concentrate on the glow model [1-2] of broken QCD. SU(3) color is spontaneously broken to SO(3)glow via a color 27 of Higgs (which is contained in the adjoint $\mathrm{x}$ adjoint of GUTs such as $\mathrm{SU}(5), \mathrm{SO}(10)$ or $\mathrm{E}(6))$. Five of the eight $\mathrm{QCD}$ gluons acquire a mass which would have to be less than $100 \mathrm{MeV}$ in order not to violate various experimental constraints. The remaining three gluons remain massless and provide a confining force for $\mathrm{SO}(3)$ glow nonsinglets. In contrast to a single gluon or a single quark, which are glow nonsinglets and thus are confined, a diquark in an $\mathrm{SU}(3)$ color 6 has a SO(3)glow singlet and, thus, can be free. Zweig [4] discussed the diquark catalysis of fusion in 1978, in particular, the $\mathrm{d}+\mathrm{d}$ fusion process (1). A stable anti-diquark state $\underline{\mathrm{u} u}$ (which we define as $Q$ ) was required since the electric charge $Z_{Q}=-4 / 3$ greatly enhances the formation of the ddQ molecule (1). The glow model specifically has diquarks as the lowest state of a free fractionally charged quark system. Since the mass of an up quark is less than that of a down quark, we expect the most stable anti-diquark is indeed the $\mathrm{Q}$.

In our scenario, the properties of the $\mathrm{Q}$ are: a) $\mathrm{Z}_{\mathrm{Q}}=-4 / 3$; b) $\mathrm{Q}$ is stable; c) The $\mathrm{Q}$ mass $\mathrm{m} \mathrm{Q}$ is roughly some few $\mathrm{GeV}$; and d) The interaction of a quark with another quark is the usual linear confining potential (slope $1 \mathrm{GeV} / \mathrm{f}$ ) out to a few $\mathrm{f}$, then falling exponentially to 0 ; this, then, provides a strong, short-range repulsive interaction barrier for $\mathrm{Q}$ interactions with ordinary matter. (Just as it is very difficult to produce free quarks, it is very difficult to get them back together.) It was argued on the basis of property d) that reaction (1i) due to its two body phase space dominates over (1ii) and (1iii) by up to a factor of $10^{5}$ (this is to be contrasted to $\mu$ catalysis of $d+d$ fusion where the corresponding reaction (1i) is negligible since the $\mu$ has no strong interaction). A further quite important 
consequence of $d$ ) is that the $Q$ will not be bound to the ${ }^{3} \mathrm{He}$ in (1ii) or the $t$ in (1iii) as is the case in a fraction of the $\mu$ catalyses which then terminate the catalysis process.

Relevant to the present ideas, it was suggested [2] that the production of free quarks would be greatly enhanced in relativistic heavy-ion collisions as compared to elementary particle collisions. In order for the resultant (non-equilibrium) quark-gluon plasma to greatly enhance the separation of fractional charge, the size of the plasma should be greater than $2 \times$ "breaking scale" of QCD which we expect must be greater than $3 \mathrm{f}$. Thus the plasma should be $>6 \mathrm{f}$. Further, the quark-gluon plasma is a crucial factor in the glow model for the formation of the diquarks. Therefore, high energy $\mathrm{Pb}-\mathrm{Pb}$ collisions should provide a tremendously exciting new environment for $\mathrm{Q}$ production that is not at all in conflict with the accurate bounds set in higher energy elementary particle collisions.

\section{EXPERIMENTAL DESIGN}

The experimental design (Fig. 1) is quite straightforward. The major part of the experiment is a cylindrical dewar perhaps $15 \mathrm{~cm}$ internal diameter and $1 \mathrm{~m}$ long (in the beam direction) with an inner wall separating it into a $50 \mathrm{~cm}$ part containing liquid $\mathrm{D}_{2}$ and a $50 \mathrm{~cm}$ part containing liquid $\mathrm{H}_{2}$ for background calibration. Windows would allow light to get out. PMTs will collect the light produced as described above during the series of $\mathrm{Q}$ catalyzed fusions in the $\mathrm{D}_{2}$ or from background events. As the electrons and ions recombine some "visible" light will be emitted which we will detect with PMTs. The key point is that we expect that there will be a lengthy series of such $\mathrm{Q}$ catalyzed fusions persisting beyond the background events before the $Q$ hits a wall via a random walk and is captured by a heavy element stopping the catalysis.

A number of the features below are rough estimates, and will be investigated in detail and optimized. We met in June with four CERN scientists who gave us very useful comments and information concerning details of this proposed experiment. This Letter of Intent incorporates these important details. 
Beam: We would request a bunched $\mathrm{Pb}$ beam, of the highest energy and highest intensity available, having a (roughly) $2 \mu \mathrm{s}$ on time and an off time of (roughly) $60 \mu \mathrm{s}$. (Karel Cornelis indicated that this time bunching is possible.) We would request a run of a day.

PMTs \& DAQ: We would have 10 PMTs, five acquiring data from each longitudinal segment of the dewar. Data would be read out every $2 \mu \mathrm{s}$. No signals would be recorded during the $2 \mu \mathrm{s}$ beam on time and for $5 \mu \mathrm{s}$ afterwards. A pedestal subtraction by hardware would reduce the data needed to be stored by a (conservative) factor of 0.05 . This gives a data rate of less than 2 Megabytes/s which can be easily stored on an inexpensive commercial tape drive. The data from a day's run could be stored on 10 tapes.

Signal: We are in the process of studying (using a PMT) the light produced in liquid $\mathrm{N}_{2}$ by $4 \mathrm{Mev}$ alpha particles from a source placed in the $\mathrm{N}_{2}$. This will be followed with the necessary studies in liquid $\mathrm{H}_{2}$. We expect the "visible" (including some UV and IR) light from the recombination of electrons and ions produced by the stopping alphas will be larger in liquid $\mathrm{H}_{2}$ since it is a simpler system than $\mathrm{N}_{2}$ with more transitions in the visible and readily detectable by the PMTs.

Dewar: The dimensions of the cylindrical dewar will be about $15 \mathrm{~cm}$ internal diameter and $1 \mathrm{~m}$ long (in the beam direction) with an inner wall separating it into a $50 \mathrm{~cm}$ part containing liquid $\mathrm{D}_{2}$ and a $50 \mathrm{~cm}$ part containing liquid $\mathrm{H}_{2}$ for background calibration. Ten windows would allow light to get out to the PMTs. We prefer the dewar to be constructed of aluminized mylar (supported by low density foam for insulation and support). The choice of mylar over glass would minimize (due to low $\mathrm{Z}$ ) possible background light (see below) from the primary and secondary beam particles exciting the walls of the dewar. We would mount the dewar and the PMTs on a rotatable support so that it could be rotated (by remote control) $180^{\circ}$ halfway through the experiment to interchange the position of the $\mathrm{D}_{2}$ and $\mathrm{H}_{2}$ relative to the target. We would request that CERN build the dewar to ensure that the safety aspects meet all CERN 
requirements. (If more convenient, two separate dewars could be built for the liquids rather than the one compartmentalized one.)

Sensitivity: Monte Carlo of Qs and Background: Our present rough calculations show that a reasonable fraction of produced Qs would stop away from the walls in the dewar and then proceed to catalyze the ddQ fusion reactions for a considerable time length ( $>10$ muon lifetimes or $20 \mu \mathrm{s})$. The two main expected backgrounds are i) large number of muons from stopping pions and ii) the beam and secondaries would excite the walls of the dewar and could produce background light. A Monte Carlo calculation will be done for i) and measurements at accelerators are being investigated to help determine ii). The effect of ii) will be minimized if the dewar is made using mylar walls (see above under Dewar). We estimate that the expected sensitivity of this proposed experiment is such that we could detect roughly one $Q$ in $10^{5}$ produced. Monte Carlo calculations will refine this number.

Resources: We would provide the data acquisition equipment needed. This will include PMTs, power supplies, tape drives, and modules to interface between the PMTs and the tape drives. The subsequent (very straightforward) data analysis will take place off-site at our computer facilities. As noted above (see above under Dewar), we would request that CERN build the dewar to ensure that the safety aspects meet all CERN requirements. (If more convenient, two separate dewars could be built for the liquids rather than the one compartmentalized one.) We also request that CERN provide the liquid $\mathrm{D}_{2}$ and $\mathrm{H}_{2}$.

Schedule: If this proposal is approved now, we anticipate being ready at the beginning of the heavy-ion beam run in the Fall, 1998. We expect that the subsequent data analysis would be completed in a very short time.

Conclusion: If a positive result is found, a follow-up experiment could examine and determine details of the time series of the $\mathrm{Q}$ induced fusion allowing one to begin to deduce the strong interaction dynamics of the $\mathrm{Q}$. Again this is a speculative endeavor, but the significance of $Q$ detection (especially via catalyzed fusion) is enormous. 
Acknowledgments: We would like to thank Karl Cornelis, Konrad Elsener, Lau Gatignon and Peter Sonderegger for very helpful discussions.

\section{References}

1. R Slansky, T Goldman \& GL Shaw, Phys Rev. Lett 47 (1981) 887

2. GL Shaw \& R Slansky, Phys. Rev. Lett. 50 (1983) 1967

3. GL Shaw \& J Tien Phys. Rev. D47 (1993) 5075

4. G Zweig, Science 201 (1978) 973

5. GL Shaw et al., Il Nuovo Cimento 102A (1989) 1441

6. LW Alvarez et al., Phys. Rev. 105 (1957) 1127

7. SE Jones, Nature 321 (1986) 127

8. GS LaRue, JD Phillips \& WM Fairbank, Phys. Rev. Lett. 47 (1981) 967

9. M Marinelli \& G Morpurgo, Phys. Rep. 85 (1982) 161

10. CL Hodges et al., Phys. Rev Lett. 47 (1981) 1651

11. MA Lindgren et al., Phys. Rev. Lett 51 (1983) 1621

12. D Liebowitz, M Binder \& KOH Ziock, Phys. Rev. Lett 50 (1983) 1540

13. PF Smith et al., Phys. Lett. B153 (1985) 188

14. GL Shaw et al., Phys. Rev. D36 (1987) 3533

15. HS Matis et al., Phys. Rev. D39 (1989) 1851

16 CD Hendricks et al., Measurement Scientific Technol. 5 (1994) 337

17. NM Mar et al., Phys. Rev D53 (1996) 6017

18 A De Rujula, RC Giles \& RL Jaffe, Phys. Rev. D17 (1978) 285

Figure 1. Schematic representation of the experimental design. The $\mathrm{Pb}$ beam hits a $\mathrm{Pb}$ target. The dewar, containing liquid $\mathrm{D}_{2}$ and liquid $\mathrm{H}_{2}$ in separate compartments, is down stream of the beam and target. 


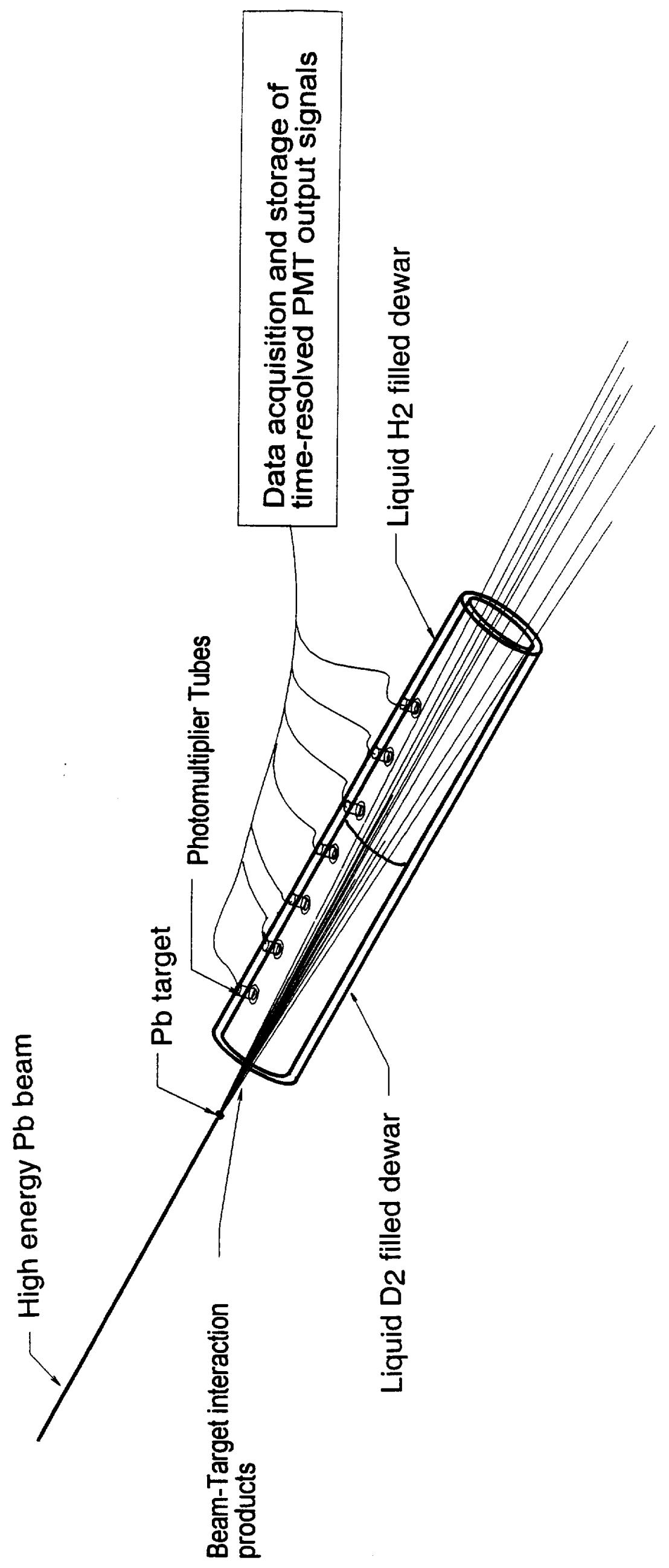

